\title{
Non-isothermal bubble rise: Non-monotonic dependence of surface tension on temperature
}

\author{
M. K. Tripathi, K. C. Sahu, G. Karapetsas**, \\ K. Sefiane ${ }^{\dagger \dagger}$, and O. K. Matar ${ }^{\dagger \dagger}$ \\ Department of Chemical Engineering, Indian Institute of Technology Hyderabad, \\ Yeddumailaram 502 205, Andhra Pradesh, India, \\ ** Department of Mechanical Engineering, University of Thessaly, Volos 38334, Greece, \\ ${ }^{\dagger}$ Department of Chemical Engineering, Imperial College London, London, SW7 2AZ, UK, \\ ${ }^{\dagger \dagger}$ School of Engineering, University of Edinburgh, Edinburgh, EH9 3JL, UK \\ (Received ?; revised ?; accepted ?. - To be entered by editorial office)
}

We study the motion of a bubble driven by buoyancy and thermocapillarity in a tube with a non-uniformly-heated walls, containing a so-called "self-rewetting fluid"; the surface tension of the latter exhibits a parabolic dependence on temperature with a well-defined minimum. In the Stokes flow limit, we derive the conditions under which a spherical bubble can come to rest in a self-rewetting fluid whose temperature varies linearly in the vertical direction, and demonstrate that this is possible for both positive and negative temperature gradients. This is in contrast to the case of simple fluids whose surface tension decreases linearly with temperature for which bubble motion is arrested for negative temperature gradients only. In the case of self-rewetting fluids, we propose an analytical expression for the position of bubble arrestment as a function of other dimensionless numbers. We also perform direct numerical simulation of axisymmetric bubble motion in a fluid whose temperature increases linearly with vertical distance from the bottom of the tube; this is done for a range of Bond and Gallileo numbers, and for various parameters that govern the functional dependence of surface tension on temperature. We demonstrate that bubble motion can be reversed and then arrested in self-rewetting fluids only, and not in linear ones, for sufficiently small Bond numbers. We also demonstrate that considerable bubble elongation is possible under significant wall confinement, and for strongly self-rewetting fluids and large Bond numbers. The mechanisms underlying the phenomena observed are elucidated by considering how the surface tension dependence on temperature affects the thermocapillary stresses in the flow.

Key words: Bubble flow, Self-rewetting fluid, Thermocapillary migration, Marangoni

\section{Introduction}

The variation in temperature of a liquid-gas interface results in the formation of surface tension gradients which induce tangential stresses, known as Marangoni stresses, driving flow in the vicinity of the interface. This mechanism is always present in non-isothermal interfacial flows and can be important in a great variety of technological applications. A characteristic problem where thermal Marangoni stresses play a significant role is the

$\dagger$ Email address for correspondence: o.matar@imperial.ac.uk 
thermocapillary migration of drops and bubbles. Much of the work in this field has been reviewed by Subramanian (1992) and Subramanian et al. (2002).

The first reported study on the thermal migration of bubbles can be found in the pioneering work of Young et al. (1959). These authors conducted experiments on air bubbles in a viscous fluid heated from below and showed that under the effect of the induced Marangoni stresses small bubbles move downwards, whereas larger bubbles move in the opposite direction as buoyancy overcomes the effect of thermocapillarity. Young et al. (1959) also provided a theoretical description of the bubble motion assuming a spherical shape and creeping flow conditions and were able to derive an analytical expression for the terminal velocity. Following this work, a series of theoretical analyses took into account the effect of convective heat transfer in the limit of both small and large Reynolds numbers (Subramanian 1981, 1983; Balasubramaniam \& Subramaniam 1996; Balasubramaniam \& Subramanian 2000; Crespo et al. 1998). Balasubramaniam \& Chai (1987) showed that the solution of Young et al. (1959) is an exact solution of the momentum equation for arbitrary Reynolds number, provided that convective heat transfer is negligible. These authors also calculated the small deformations of a drop from a spherical shape.

The main motivation for the aforementioned studies came from microgravity applications and buoyancy was considered to be negligible. The effect of combined action of buoyancy and thermocapillarity was studied by Merritt et al. (1993) employing numerical simulations. Balasubramaniam (1998) presented an asymptotic analysis in the limiting case of large Reynolds and Marangoni numbers, including the buoyant contribution as wells as a temperature varying viscosity. It was shown that the steady migration velocity, at leading order, is a linear combination of the velocity for purely thermocapillary motion and the buoyancy-driven rising velocity. Later, Zhang et al. (2001) performed a theoretical analysis for small Marangoni numbers under the effect of gravity and showed that inclusion of inertia is crucial in the development of an asymptotic solution for the temperature field. The asymptotic analysis presented by these authors is based on the assumption of a finite velocity and cannot be used for the case of a stationary bubble. The latter problem has to be analyzed separately as was done by Balasubramaniam \& Subramanian (2004). The solution of this problem is complicated by the presence of a singularity failing to satisfy the far-field condition. Yariv \& Shusser (2006) introduced an exponentially small artificial bubble velocity as a regularization parameter to account for the inability of the asymptotic expansion to satisfy the condition of exact bubble equilibrium. They were able to evaluate the correction for the hydrodynamic force exerted on the bubble including convective heat transfer; this correction was shown to be independent of the regularization parameter.

A great variety of numerical methods have been proposed in order to take into account the effect of surface deformation. These range from boundary-fitted grids (Chen \& Lee 1992; Welch 1998), to the level-set method (Haj-Hariri et al. 1997; Zhao et al. 2010), the VOF method (Ma \& Bothe 2011), diffuse-interface methods (Borcia \& Bestehorn 2007) and hybrid schemes of the Lattice-Boltzmann and the finite difference method (Liu et al. 2013). It was shown by Chen \& Lee (1992) that surface deformation of gas bubbles reduces considerably their terminal velocity. The same effect was found also in the case of viscous drops by Haj-Hariri et al. (1997). Later, Welch (1998) demonstrated that as the capillary number increases and the bubble deformation becomes important, the bubbles do not reach a steady state terminal velocity. As was shown by Herrmann et al. (2008), the assumption of quasi-steady-state is not valid also for large Marangoni numbers. The latter finding was very recently confirmed by Wu \& Hu $(2012,2013)$.

Most of the studies mentioned above concern the motion of a single bubble or drop 
in an unconfined medium. Acrivos et al. (1990) studied systems of multiple drops in the creeping flow limit and showed that the drops do not interact. However, when inertial effects are included it was shown by Nas \& Tryggvason (2003) and Nas et al. (2006) that there are strong interactions between the droplets. The thermocapillary interaction between spherical drops in the creeping flow limit was discussed by several authors (Leshansky et al. 2001; Leshansky \& Nir 2001). In the vicinity of a solid wall, the drop migration velocity is affected by the hydrodynamic resistance due to the presence of the wall as well as by the thermal interaction between the wall and the drop. Meyyappan \& Subramanian (1987) examined the motion of a gas bubble close to a rigid surface with an imposed far-field temperature gradient and found that the surface exerts weaker influence in the case of parallel motion than in the case of motion normal to it. Keh et al. (2002) investigated the motion of a spherical drop between two parallel plane walls and found that the wall effect could speed up or slow down the droplet depending on the thermal conductivity of the droplet and the imposed boundary conditions at the wall. Chen et al. (1991) considered the case of a spherical drop and studied the thermocapillary migration inside an insulated tube with an imposed axial temperature gradient. They found that the migration velocity in the tube never exceeds the value in an infinite medium due to the hydrodynamic retarding forces that are being developed.

Very recently, Mahesri et al. (2014) extended the work of Chen et al. (1991) to take into account the effect of interfacial deformation. It was found that as in the case of the spherical drop the migration velocity of the confined drop is always lower than that of an unbounded drop. Brady et al. (2011) presented numerical simulations of a droplet inside a rectangular box and showed that for low Marangoni numbers the drop rapidly settles to a quasi steady state whereas for high Marangoni numbers the initial conditions affect significantly the behaviour of the droplet. In the case of severe confinement inside a tube, the drop can become quite long. Such a case was studied by Hasan \& Balasubramaniam (1989) and Wilson (1993) who focused their attention on the thin film region away from the drop ends, and were able to derive a relation between the migration velocity and the film thickness. Later, Mazouchi \& Homsy (2000, 2001) used lubrication theory to determine the liquid film thickness and migration velocity for the case of a cylindrical and polygonal tube, respectively.

It is well known that the surface tension of common fluids, such as air, water, and various oils, decreases almost linearly with increasing temperature; all of the abovementioned studies have considered such fluids. In the present paper, we are interested in the thermocapillary migration of a deformable bubble inside a cylindrical tube filled with liquids that exhibit a non-monotonic dependence of the surface tension on temperature. In particular, these so-called "self-rewetting" fluids (Vochten \& Petre 1973; Petre \& Azouni 1984; Limbourgfontaine et al. 1986; Savino et al. 2009, 2013), which are non-azeotropic, high carbon alcohol solutions, have quasi parabolic surface tensiontemperature curves with well-defined minima; the parabolicity of these curves increases with alcohol concentration. These fluids were first studied by Vochten \& Petre (1973) who observed the occurrence of the minimum in surface tension with temperature in high carbon alcohol solutions. Petre \& Azouni (1984) carried out experiments that involved imposing a temperature gradient on the surface of alcohol aqueous solutions, and used talc particles to demonstrate the unusual behaviour of these fluids. Experimental work on these fluids was also carried out under reduced-gravity conditions by Limbourgfontaine et al. (1986). The term "self-rewetting" was coined by Abe et al. (2004) who studied the thermophysical properties of dilute aqueous solution of high carbon alcohols. Due to thermocapillary stresses, and the shape of the surface tension-temperature curve, the 
fluids studied spread "self-rewet" by spreading spontaneously towards the hot regions, thereby preventing dry-out of hot surfaces and enhancing the rate of heat transfer.

Due to the abovementioned properties, "self-rewetting" fluids were shown to be associated with substantially higher critical heat fluxes in heat pipes compared to water (Suzuki et al. 2005; Mcgillis \& Carey 1996; S. Ahmed \& Carey 1999). Savino et al. (2009) illustrated the anomalous behaviour of self-rewetting fluids by performing experiments to visualise the behaviour of vapour slugs inside wickless heat pipes made of pyrex borosilicate glass capillaries. They found that the size of the slugs was considerably smaller than that associated with fluids such as water. More recently, work on self-rewetting fluids was extended to microgravity conditions for space applications on the International Space Station. Savino et al. (2013), and Hu et al. Hu et al. (2014) demonstrated that the use of these fluids within micro oscillating heat pipes led to an increase in the efficiency of these devices. In a slightly different context, it was very recently shown that the presence of a minimum in surface tension can also have a significant impact on the dynamics of the flow giving rise to very interesting phenomena such as the thermally induced "superspreading" (Karapetsas et al. 2014).

In this paper, we study the buoyancy-driven rise of a bubble inside a tube imposing a constant temperature gradient along the wall. To account for the non-monotonicity of surface tension we consider a quadratic dependence on temperature. We examine the Stokes flow limit first and derive conditions under which the motion of a spherical bubble can be arrested in self-rewetting fluids even for positive temperature gradients (in the opposite direction of gravity). We then employ a diffuse-interface method (Ding et al. 2007) to follow the deforming bubble along the domain in the presence of inertial contributions. Our results indicate that for self-rewetting fluids, the bubble motion departs considerably from the behaviour of ordinary fluids and the dynamics may become complex as the bubble crosses the position of minimum surface tension. As will be shown below, under certain conditions, the motion of the bubble can be reversed, and then arrested, or the bubble can become elongated significantly.

The rest of the paper is organized as follows. In Section II, we outline the governing equations, and in Section III present our analytical results for a spherical bubble in the Stokes flow limit. We then discuss our numerical results for deformable bubbles in the presence of inertia in Section IV. Finally, concluding remarks are given in Section V.

\section{Formulation}

\subsection{Governing equations}

We consider the motion of an axisymmetric gas bubble of fluid ' $\mathrm{B}$ ' and initial radius $R$ under the action of buoyancy inside a cylindrical tube of diameter $H$ and height $L$, as shown in Fig. 1. The tube is filled with an incompressible, Newtonian fluid 'A'; below, we shall take the properties of fluids $\mathrm{A}$ and $\mathrm{B}$ to reflect those of a liquid and a gas, respectively. We use cylindrical coordinates $(r, z)$ to model the axisymmetric bubble dynamics starting from a position $z_{i}$. Thus the flow dynamics is assumed to be symmetrical about $r=0$. The rigid and impermeable wall is located at $r=H / 2$. The acceleration due to gravity, $g$ is acting in the negative $z$ direction, as shown in Fig. 1.

The equations of mass, momentum and energy conservation which govern the flow can be respectively written as:

$$
\begin{gathered}
\nabla \cdot \mathbf{u}=0 \\
\rho\left[\frac{\partial \mathbf{u}}{\partial t}+\mathbf{u} \cdot \nabla \mathbf{u}\right]=-\nabla p+\nabla \cdot\left[\mu\left(\nabla \mathbf{u}+\nabla \mathbf{u}^{T}\right)\right]+\mathbf{F},
\end{gathered}
$$




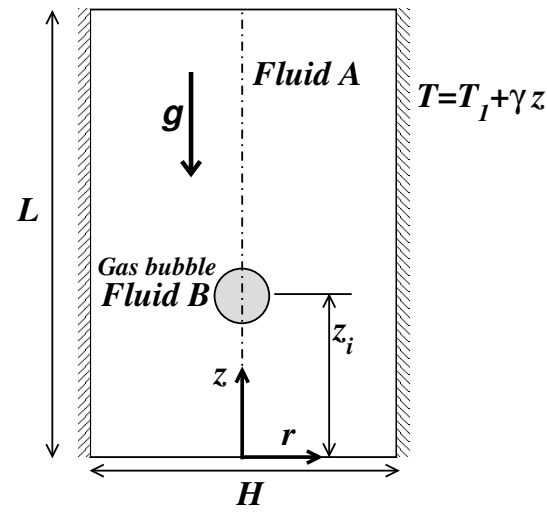

Figure 1: Schematic diagram of a bubble moving inside a Newtonian fluid under the action of buoyancy. The initial location of the bubble is at $z=z_{i}$; unless specified, the value of $H, L$ and $z_{i}$ are $6 R, 48 R$, and $10.5 R$, respectively. The acceleration due to gravity, $g$, acts in the negative $z$ direction.

$$
\frac{\partial T}{\partial t}+\mathbf{u} \cdot \nabla T=\nabla \cdot(\alpha \nabla T)
$$

where $\mathbf{u}, p$ and $T$ denote the velocity, pressure, and temperature fields of the fluid, respectively, $t$ represents time; $\mathbf{F}$ is the body and surface forces, such as the gravity and surface tension force per unit volume, and $\alpha$ denotes the thermal diffusivity. For the volume fraction, $c$, which takes on values between 0 and 1 for the gaseous and liquid phases, respectively, we have the following equation:

$$
\frac{\partial c}{\partial t}+\mathbf{u} \cdot \nabla c=0
$$

In order to model the behaviour of a self-rewetting fluid, we use the following relationship for the functional dependence of the surface tension on temperature:

$$
\sigma=\sigma_{0}-\beta_{1}\left(T-T_{1}\right)+\beta_{2}\left(T-T_{1}\right)^{2},
$$

where $\beta_{1} \equiv-\left.\frac{d \sigma}{d T}\right|_{T_{1}}$ and $\left.\beta_{2} \equiv \frac{1}{2} \frac{d^{2} \sigma}{d T^{2}}\right|_{T_{1}}$. A linear temperature variation is imposed in the vertical direction with a constant gradient $\gamma$, and Fig. 2 shows the corresponding variation in $\sigma$ with $z$ for different values of $\beta_{1}$ and $\beta_{2}$. As can be seen from this figure, the parabolic dependence of $\sigma$ on $z$ becomes more pronounced, with a deeper minimum, located at $z=z_{m}$, for increasing $\beta_{1}$ and $\beta_{2}$; this is expected to alter the type of Marangoni flow observed in case fluids that exhibit a simple linear variation of $\sigma$ with $T$ which we will refer to in this paper as 'linear' fluids. Below, we will explore the dynamics of the bubble as it rises starting from $z_{i}$, which may be either below or above $z=z_{m}$, for both linear and self-rewetting fluids. The dependence of this dynamics on $\beta_{1}$ and $\beta_{2}$, which parameterise the behaviour of various self-rewetting fluids, will also be studied.

The viscosity is assumed to depend on the temperature and the volume fraction as follows:

$$
\mu=c \mu_{A} e^{-\left(\frac{T-T_{1}}{T_{m}-T_{1}}\right)}+(1-c) \mu_{B}\left\{1+\left(\frac{T-T_{1}}{T_{m}-T_{1}}\right)^{3 / 2}\right\},
$$

where $T_{1}$ and $T_{m}$ are the temperature at the bottom of the tube $(z=0)$ and temperature at $z=z_{m} ; \mu_{A}$ and $\mu_{B}$ are the viscosity of the liquid and gas at temperature $T_{1}$, respectively. This viscosity dependence on temperature for the liquid and gaseous phases 


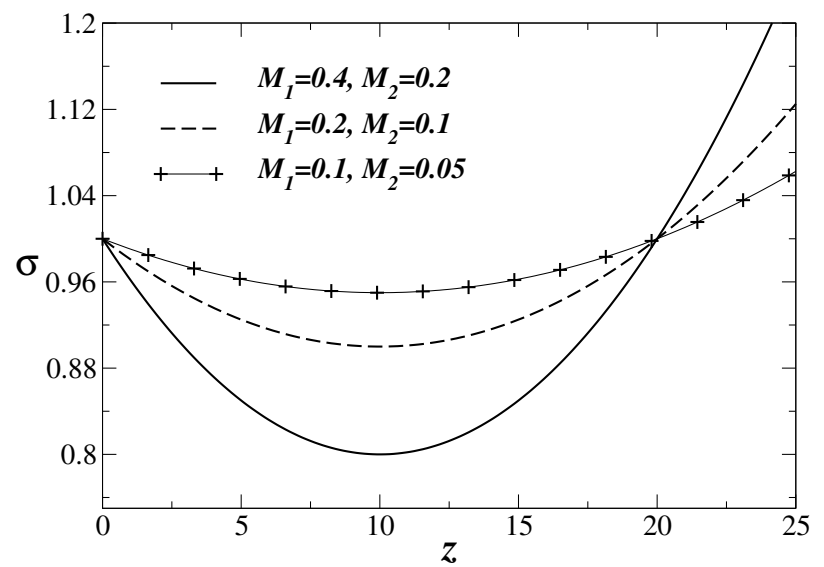

Figure 2: Variation of the liquid-gas surface tension along the wall of the tube for $\Gamma=0.1$ and various values of $M_{1}$ and $M_{2}$.

are taken from Nahme (1940). The density is given by

$$
\rho=\rho_{A} c+\rho_{B}(1-c),
$$

while the thermal diffusivity is expressed by

$$
\alpha=\alpha_{A} c+\alpha_{B}(1-c)
$$

in which $\rho_{A}$ and $\rho_{B}$ denote the density, and $\alpha_{A}$ and $\alpha_{B}$ represent the thermal diffusivity of the liquid and gas phases, respectively.

\subsection{Scaling}

The following scaling is employed to render the governing equations dimensionless:

$$
\begin{gathered}
(r, z)=R(\widetilde{r}, \widetilde{y}), t=\frac{R}{V} \widetilde{t}, \mathbf{u}=V \widetilde{\mathbf{u}}, p=\rho_{A} V^{2} \widetilde{p} \\
\mu=\mu_{A} \widetilde{\mu}, \rho=\rho_{A} \widetilde{\rho}, \alpha=\alpha_{A} \widetilde{\alpha}, T=\widetilde{T}\left(T_{m}-T_{1}\right)+T_{1}, \\
\sigma=\sigma_{0} \widetilde{\sigma}, \beta_{1}=\frac{\sigma_{0}}{T_{m}-T_{1}} M_{1}, \beta_{2}=\frac{\sigma_{0}}{\left(T_{m}-T_{1}\right)^{2}} M_{2}, \gamma=\frac{\left(T_{m}-T_{1}\right)}{R} \Gamma,
\end{gathered}
$$

where the velocity scale is $V=\sqrt{g R}, \sigma_{0}$ is the surface tension at $T_{1}$, and the tildes designate dimensionless quantities. After dropping tildes from all non-dimensional terms, the governing dimensionless equations are given by

$$
\begin{gathered}
\nabla \cdot \mathbf{u}=0 \\
\frac{\partial \mathbf{u}}{\partial t}+\mathbf{u} \cdot \nabla \mathbf{u}=-\nabla p+\frac{1}{\mathrm{Ga}} \nabla \cdot\left[\mu\left(\nabla \mathbf{u}+\nabla \mathbf{u}^{T}\right)\right]+\mathbf{F}, \\
\frac{\partial T}{\partial t}+\mathbf{u} \cdot \nabla T=\frac{1}{\mathrm{GaPr}} \nabla \cdot(\alpha \nabla T), \\
\frac{\partial c}{\partial t}+\mathbf{u} \cdot \nabla c=0,
\end{gathered}
$$

where $\mathrm{Ga} \equiv \rho_{A} V R / \mu_{A}$ denotes the Galileo number; $\operatorname{Pr} \equiv c_{p} \mu_{0}\left(T_{1}\right) / \lambda_{A}$ is the Prandtl number, wherein $c_{p}$ is the specific heat capacity of the surrounding fluid, and $\lambda_{A}$ is 
the thermal conductivity of the liquid. The dimensionless viscosity, $\mu$, has the following dependence on $T$ and $c$ :

$$
\mu=c e^{-T}+(1-c) \mu_{r}\left(1+T^{3 / 2}\right),
$$

where $\mu_{r} \equiv \mu_{B} / \mu_{A}$ is the viscosity ratio. The dimensionless density and thermal diffusivity are given by (Haj-Hariri et al. 1997)

$$
\begin{gathered}
\rho=c+\rho_{r}(1-c), \quad \text { and } \\
\alpha=c+\alpha_{r}(1-c),
\end{gathered}
$$

respectively, wherein $\rho_{r} \equiv \rho_{B} / \rho_{A}$ and $\alpha_{r} \equiv \alpha_{B} / \alpha_{A}$ which correspond to the density and thermal diffusivity ratios, respectively. In Eq. (2.11), the force $\mathbf{F}$ is given by (continuum surface force formulation (Brackbill et al. 1992))

$$
\mathbf{F}=\frac{\kappa \delta_{s}}{B o}\left[1-M_{1} T+M_{2} T^{2}\right] \mathbf{n}-\rho \mathbf{j},
$$

in which the first and second terms on the right-hand-side correspond to the capillary and gravitational contributions, respectively. In the former, the dependence of $\sigma$ on $T$, using Eq. (2.5) has been included. In Eq. (2.17), j represents the unit vector in the vertical direction, $\kappa$ is the curvature of the interface calculated as the divergence of the unit normal to the interface, $\mathbf{n}, \delta_{s}$ is the Dirac distribution function in the vicinity of the interface, and $B o \equiv \rho_{A} g R^{2} / \sigma_{0}$ is the Bond number. A discussion of the results is presented next.

\section{Analytical results: Stokes flow limit}

In this section, we provide a discussion of our analytical results. We derive expressions for the terminal velocity of a spherical bubble rising vertically through a quiescent and unconfined liquid in the Stokes flow limit in which thermocapillary stresses arise due to a temperature gradient imposed on the liquid. For the purpose of this calculation we will ignore the presence of walls and consider the case of unconfined flow. We show how the dependence of the surface tension on temperature, represented by Eq. (2.5), affects the terminal bubble speed, and the magnitude and sign of the temperature gradient required to arrest bubble motion; this is also contrasted with the case of a linear fluid.

We adopt a spherically-symmetric coordinate system, $(r, \theta)$, with the polar angle, $\theta$, measured from the bottom of the bubble $(\theta=0)$ to the top of the bubble $(\theta=\pi)$; $\mathbf{u}=u_{r} \mathbf{i}_{r}+u_{\theta} \mathbf{i}_{\theta}$ is the velocity field in which $u_{r}$ and $u_{\theta}$ represent its radial and azimuthal components, and $\mathbf{i}_{r}$ and $\mathbf{i}_{\theta}$ denote the unit vectors in the $r$ and $\theta$ directions, respectively. The $z$-axis originates at the bubble centre and is oriented vertically upwards so that it coincides with the axis of symmetry of the bubble. The unit vector in the $z$-direction is expressed by $\mathbf{i}_{z}=-\eta \mathbf{i}_{r}+\left(1-\eta^{2}\right)^{1 / 2} \mathbf{i}_{\theta}$ in which $\eta \equiv \cos \theta$. Note that we have adopted a different coordinate system from that in Section 2 temporarily for the purpose of this calculation and redefined the origin of the $z$-axis. At the end of the present subsection, we shall revert to the use of cylindrical coordinates. We also adopt a frame of reference that moves with the centre of the bubble, which is scaled on the steady translational speed of the bubble, $U$; this speed will be determined as part of the solution. Note that all quantities presented in this subsection are in dimensional terms.

We assume that heat transfer is dominated by conduction so that the temperature field in the liquid, $T$, is governed by

$$
\nabla^{2} T=0
$$


We impose a linear temperature distribution in the liquid, so that at large distances from the bubble we have

$$
T_{\infty}(z)=T_{\infty}(0)+\gamma z^{\prime} .
$$

Here, $T_{\infty}(0)$ denotes the temperature at $z^{\prime}=0$, the position of the centre of the bubble. At the bubble surface, $r=R$, we demand continuity of the thermal flux:

$$
\frac{\partial T}{\partial r}=\left(\frac{\lambda_{B}}{\lambda_{A}}\right) \frac{\partial T_{g}}{\partial r}
$$

where $\lambda_{B}$ denotes the thermal conductivity of the gas. We assume that $\lambda_{A} \gg \lambda_{B}$ so that Eq. (3.3) reduces to

$$
\frac{\partial T}{\partial r}=0 .
$$

The general solution of Eq. (3.1) is given by

$$
T=\sum_{n=0}^{\infty}\left[A_{n}\left(\frac{r}{R}\right)^{n}+B_{n}\left(\frac{r}{R}\right)^{-(n+1)}\right] P_{n}(\eta),
$$

where $P_{n}(\eta)$ are Legendre polynomials of the first kind of degree $n$. We apply the no-flux condition given by Eq. (3.4) at $r=R$ :

$$
B_{n}=\left(\frac{n}{n+1}\right) A_{n}
$$

Substitution of Eq. (3.6) into Eq. (3.5) gives

$$
T=\sum_{n=0}^{\infty} A_{n}\left[\left(\frac{r}{R}\right)^{n}+\frac{n}{n+1}\left(\frac{r}{R}\right)^{-(n+1)}\right] P_{n}(\eta) .
$$

To match to the far field condition, we set $z^{\prime}=r \cos (\pi-\theta)=-r \cos \theta=-r \eta$ in Eq. (3.2) so that $T_{\infty}(z)=T_{\infty}(0)-\gamma r \eta$. Matching this equation to Eq. (3.7) yields

$$
A_{0}=T_{\infty}(0), \quad A_{1}=-\gamma R, \quad A_{n}=0 \text { for } n \geqslant 2 .
$$

Substitution of these values into Eq. (3.7) gives

$$
T=T_{\infty}(0)-\gamma r\left[1+\frac{1}{2}\left(\frac{r}{R}\right)^{-3}\right] \eta .
$$

The solution for the velocity field in the fluid, $\mathbf{u}=\left(u_{r}, u_{\theta}\right)$, is subject to the following boundary conditions:

$$
\begin{gathered}
\mathbf{u} \rightarrow-\mathbf{i}_{z} \quad \text { as } \quad|\mathbf{r}| \rightarrow \infty, \\
u_{r}=0 \quad \text { at } \quad r=R, \\
\tau_{r \theta}+\frac{\partial \sigma}{\partial \theta}=0 \quad \text { at } \quad r=R,
\end{gathered}
$$

where $\tau_{r \theta}$ is the tangential stress.

As noted above, the flow is axisymmetric about the $z$-axis, hence the solution to the Stokes flow problem can be expressed in terms of the streamfunction, $\psi$ (Leal 1992):

$$
\psi=U R^{2}\left[-\left(\frac{r}{R}\right)^{2} Q_{1}(\eta)+\sum_{n=1}^{\infty}\left[C_{n}\left(\frac{r}{R}\right)^{2-n}+D_{n}\left(\frac{r}{R}\right)^{-n}\right] Q_{n}(\eta)\right] .
$$

This is the general solution for flow past an axisymmetric body of arbitrary shape in the 
Stokes flow limit. Here, $Q_{n}$ are integrals of $P_{n}(\eta)$, and closely related to the Gegenbauer polynomials. The polynomials relevant to the present work are

$$
Q_{1}(\eta)=\frac{1}{2}\left(\eta^{2}-1\right), \quad Q_{2}(\eta)=\frac{\eta}{2}\left(\eta^{2}-1\right) .
$$

The solution expressed by Eq. (3.13) is chosen to satisfy the following equation

$$
E^{4} \psi=0 \text {, }
$$

where $E^{2}$ is given by

$$
E^{2} \equiv \frac{\partial^{2}}{\partial r^{2}}+\frac{\left(1-\eta^{2}\right)}{r^{2}} \frac{\partial^{2}}{\partial \eta^{2}},
$$

as well as the far field condition given by Eq. (3.10). The streamfunction $\psi$ is related to $u_{r}$ and $u_{\theta}$ by

$$
u_{r}=-\frac{1}{r^{2}} \frac{\partial \psi}{\partial \eta}, \quad u_{\theta}=-\frac{1}{r\left(1-\eta^{2}\right)^{1 / 2}} \frac{\partial \psi}{\partial r} .
$$

It can be shown that for flow problems that have $\psi$ expressed as in Eq. (3.13), the component of the dimensional force along the axis of symmetry, $F_{z}$, exerted by the surrounding fluid on an axisymmetric body of arbitrary shape with its centre of mass at $|\mathbf{x}|=0$ is given by the following general formula

$$
F_{z}=4 \pi \mu_{A} U R C_{1} .
$$

At steady-state, this drag force balances the buoyancy force:

$$
4 \pi \mu_{A} U R C_{1}=\frac{4}{3} \pi\left(\rho_{A}-\rho_{B}\right) R^{3} g \approx \frac{4}{3} \pi \rho_{A} R^{3} g,
$$

where we have assumed that $\rho_{A} \gg \rho_{B}$. Equation (3.19) suggests that $C_{1}$ is the only coefficient that we need to compute in order to determine the terminal velocity of the bubble.

The no-penetration condition given by Eq. (3.11) can be re-expressed as $\partial \psi / \partial \eta=0$ at $r=R$. It follows from this condition that $\psi$ is constant at $r=R$. Since $\psi=0$ at $\theta=0$ and $\theta=\pi$, corresponding to $\eta= \pm 1$, for all $r$ because of symmetry, then Eq. (3.11) can be re-written as

$$
\psi=0 \quad \text { at } \quad r=R .
$$

Application of this condition yields

$$
0=-Q_{1}(\eta)+\sum_{n=1}^{\infty}\left(C_{n}+D_{n}\right) Q_{n}(\eta) .
$$

The tangential stress balance given by Eq. (3.12) can be re-expressed as

$$
-\mu_{A} r \frac{\partial}{\partial r}\left(\frac{1}{r^{2}\left(1-\eta^{2}\right)^{1 / 2}} \frac{\partial \psi}{\partial r}\right)+\frac{\partial \sigma}{\partial \theta}=0 \text { at } r=R .
$$

Using Eq. (2.5), the surface tension gradient, $\partial \sigma / \partial \theta$ is then given by

$$
\begin{aligned}
\frac{\partial \sigma}{\partial \theta} & =\frac{\partial \sigma}{\partial T} \frac{\partial T}{\partial \theta} \\
& =\left[-\beta_{1}+2 \beta_{2}\left(T_{\infty}(0)-T_{1}-\gamma r\left[1+\frac{1}{2}\left(\frac{r}{R}\right)^{-3}\right] \eta\right)\right] \\
& \times\left(\gamma r\left[1+\frac{1}{2}\left(\frac{r}{R}\right)^{-3}\right]\left(1-\eta^{2}\right)^{1 / 2}\right),
\end{aligned}
$$


where $T_{1}$ is a reference temperature defined in Section 2.1. Substitution of Eqs. (3.13) and (3.23) into Eq. (3.22) yields

$$
\begin{aligned}
-2 Q_{1}(\eta) & +2 C_{1} Q_{1}(\eta)-4 D_{1} Q_{1}(\eta)+\sum_{n=2}^{\infty}\left((2-n)(1+n) C_{n}-n(n+3) D_{n}\right) Q_{n}(\eta) \\
& =\frac{-3 \gamma R}{2 \mu_{A} U}\left(-\beta_{1}+2 \beta_{2}\left[T_{\infty}(0)-T_{1}-\frac{3}{2} \gamma R \eta\right]\right)\left(1-\eta^{2}\right) \\
& =\frac{-3 \gamma R \beta_{1}}{\mu_{A} U}\left[1-\frac{2 \beta_{2}}{\beta_{1}}\left(T_{\infty}(0)-T_{1}\right)\right] Q_{1}(\eta)-\frac{9 \gamma^{2} R^{2} \beta_{2}}{\mu_{A} U} Q_{2}(\eta)
\end{aligned}
$$

From Eq. (3.21) we get

$$
-Q_{1}+\left(C_{1}+D_{1}\right) Q_{1}+\sum_{n=2}^{\infty}\left(C_{n}+D_{n}\right) Q_{n}=0,
$$

whence

$$
C_{1}+D_{1}=1, \quad \text { and } \quad C_{n}=-D_{n} \text { for } n \geqslant 2
$$

For $\beta_{2}=0$, i.e. for a linear fluid, then from Eq. (3.24) we get

$$
-2 Q_{1}+2 C_{1} Q_{1}-4 D_{1} Q_{1}=-\frac{3 \gamma R \beta_{1}}{\mu_{A} U} Q_{1}, \text { and } C_{n}=\frac{n(n+3)}{(n+1)(2-n)} D_{n}, \text { for } n \geqslant 2 .
$$

Thus, we deduce that

$$
C_{1}=1-\frac{\gamma R \beta_{1}}{2 \mu_{A} U}, \quad D_{1}=\frac{\gamma R \beta_{1}}{2 \mu_{A} U}
$$

For $n \geqslant 2$,

$$
C_{n}=\frac{n(n+3)}{(n+1)(2-n)} D_{n}=-D_{n} \Rightarrow\left(\frac{n(n+3)}{(n+1)(2-n)}+1\right) D_{n}=0
$$

However, the coefficient of $D_{n}$ is not zero, so $D_{n}=C_{n}=0$ for $n \geqslant 2$. From Eq. (3.19), we arrive at the following expression for $U$, after making use of $C_{1}$ from Eq. (3.28):

$$
U=\frac{\rho_{A} R^{2} g}{3 \mu_{A}}\left(1+\frac{3}{2} \frac{\gamma \beta_{1}}{\rho_{A} R g}\right) .
$$

Thus, if $U=0$ the bubble rise is arrested provided

$$
\gamma=\gamma_{c}=-\frac{2 \rho_{A} R g}{3 \beta_{1}}
$$

This equation implies that for a linear fluid, the temperature gradient must be negative in order for the bubble to come to rest (Leal 1992).

For a self-rewetting fluid with $\beta_{2} \neq 0$, and following a similar procedure to that dis- 
cussed above, the relevant coefficients are

$$
\begin{aligned}
C_{1} & =1-\frac{\gamma R \beta_{1}}{2 \mu_{A} U}\left[1-\frac{2 \beta_{2}}{\beta_{1}}\left(T_{\infty}(0)-T_{1}\right)\right], \\
C_{2} & =-\frac{9 \gamma^{2} R^{2} \beta_{2}}{10 \mu_{A} U} \\
D_{1} & =\frac{\gamma R \beta_{1}}{2 \mu_{A} U}\left[1-\frac{2 \beta_{2}}{\beta_{1}}\left(T_{\infty}(0)-T_{1}\right)\right], \\
D_{2} & =\frac{9 \gamma^{2} R^{2} \beta_{2}}{10 \mu_{A} U}
\end{aligned}
$$

and $C_{n}=D_{n}=0$ for $n \geqslant 3$. Substitution of $C_{1}$ into Eq. (3.19) yields the following expression for the terminal velocity $U$ :

$$
U=\frac{\rho_{A} R^{2} g}{3 \mu_{A}}\left(1+\frac{3}{2} \frac{\gamma \beta_{1}}{\rho_{A} R g}\left[1-\frac{2 \beta_{2}}{\beta_{1}}\left(T_{\infty}(0)-T_{1}\right)\right]\right) .
$$

The expression for $\gamma_{c}$ that leads to bubble arrest and $U=0$ is given by:

$$
\gamma_{c}=-\frac{2}{3} \frac{\rho_{A} R g}{\beta_{1}\left(1-2 \frac{\beta_{2}}{\beta_{1}}\left(T_{\infty}(0)-T_{1}\right)\right)} .
$$

For $\beta_{2}=0$, this equation reduces to Eq. (3.31). Equation (3.37) suggests that $\gamma_{c}$ is positive (negative) if $2 \beta_{2}\left(T_{\infty}(0)-T_{1}\right) / \beta_{1}>1\left(2 \beta_{2}\left(T_{\infty}(0)-T_{1}\right) / \beta_{1}<1\right)$ in the case of a self-rewetting fluid, in contrast to the case of a linear fluid in which $\gamma_{c}<0$.

We note that if we had assumed that the temperature distribution given by Eq. (3.2) applied everywhere, including at the bubble surface, $r=R$, then the formula for the terminal velocity would have been expressed by

$$
U=\frac{\rho_{A} R^{2} g}{3 \mu_{A}}\left(1+\frac{\gamma \beta_{1}}{\rho_{A} R g}\left[1-\frac{2 \beta_{2}}{\beta_{1}}\left(T_{\infty}(0)-T_{1}\right)\right]\right),
$$

and the expression for $\gamma_{c}$ by

$$
\gamma_{c}=-\frac{\rho_{A} R g}{\beta_{1}\left(1-2 \frac{\beta_{2}}{\beta_{1}}\left(T_{\infty}(0)-T_{1}\right)\right)} .
$$

Reverting back to the coordinate system of the previous section the position of the bubble centre is given by

$$
z=\frac{T_{\infty}(0)-T_{1}}{\gamma}
$$

and using Eq. (3.37) it is possible to derive an expression for the terminal vertical position of the bubble, $z_{c}$ :

$$
z_{c}=\left(\frac{\beta_{1} \gamma_{c}}{2}+\frac{\rho_{A} R g}{3}\right) \frac{1}{\beta_{2} \gamma_{c}^{2}} .
$$

In the following section, we compare the predictions of Eq. (3.41) with those obtained from the numerical simulations. We turn our attention now to the numerical results.

\section{Numerical results}

In this section, we present a discussion of our numerical results starting with a presentation of the numerical procedures used to carry out the computations. 
12

(a)

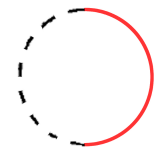

(b)

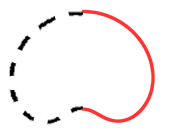

Tripathi et al.

(c)

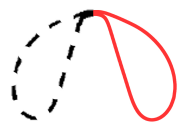

(d)

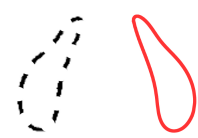

Figure 3: Comparison of the evolution of the shape of the bubble obtained from our simulation (shown by red line) with Sussman \& Smereka (1997) (dashed line) at various times in an isothermal system: (a) $t=0$, (b) $t=0.8$, (c) $t=1.6$ and (d) $t=2.4$. The parameter values are $G a=100, B o=200, \rho_{r}=10^{-3}$ and $\mu_{r}=10^{-2}$.

\subsection{Numerical method and validation}

To account for the effects of inertia and confinement we solve the governing equations numerically. For the rest of the paper we will make use of the cylindrical coordinates. We use a bespoke finite-volume flow solver (Tripathi et al. 2014) that solves Eqs. (2.10)(2.12) on a staggered grid. The scalar variables (the pressure and the volume fraction of the outer fluid) are defined at the cell-centres and the velocity components are defined at the cell faces, respectively. In our solver, weighted-essentially-non-oscillatory (WENO), and central difference schemes are used to discretize the advective and diffusive terms in Eq. (2.13), respectively. In order to achieve second-order accuracy, the Adams-Bashforth and the Crank-Nicholson methods are used to discretize the advective and dissipation terms in Eq. (2.11), respectively.

We assume that the flow is symmetric about the axis $r=0$. The no-slip and nopenetration boundary conditions are used at the walls and the Neumann boundary conditions are imposed at the top and bottom parts of the domain. It is to be noted that the density and the viscosity ratios in the problem considered in the present study, $\rho_{r}$ and $\mu_{r}$, are very large, which can create spurious currents at the interface. Thus the code has been validated against Gerris, an open-source finite-volume fluid flow solver (Popinet 2003), which minimizes this problem by using the balanced-force continuum surface force formulation for the calculation of surface tension. We also note that in some of the cases we used only Gerris. The validation of our code is presented in Fig. 3, where we compare the results obtained using our code with those obtained by Sussman \& Smereka (1997). Here, the parameters were chosen such that the numerical computation is particularly taxing, involving severe deformation of the bubble. The reader is also referred to the supplementary material of Tripathi et al. (2014) for an extensive validation of the present code. In addition, we have ensured that convergence is indeed achieved upon mesh refinement.

Below, we present a discussion of our results for the following set of 'base' parameters: $B o=10^{-2}, G a=10, H=6, \quad \mu_{r}=10^{-2}, M_{1}=0.4, \rho_{r}=10^{-3}, z_{i}=10.5$, and $\Gamma=0.1$, which are consistent with the case of a small air bubble rising in water due to buoyancy, in the presence of strong mean surface tension and Marangoni effects, and appreciable inertial contributions. We will contrast the difference in behaviour between bubble motion in linear and self-rewetting fluids by studying the effect of parameter $M_{2}$ on the dynamics.

\subsection{Discussion}

We begin the discussion of our results by showing in Fig. 4 the temporal variation of the centre of gravity, $z_{C G}$, of a rising bubble for three different cases: the isothermal case, and the cases of a simple linear fluid, and a self-rewetting one rising in a tube whose walls are heated with a linear temperature profile of constant gradient $\Gamma>0$. It can be 


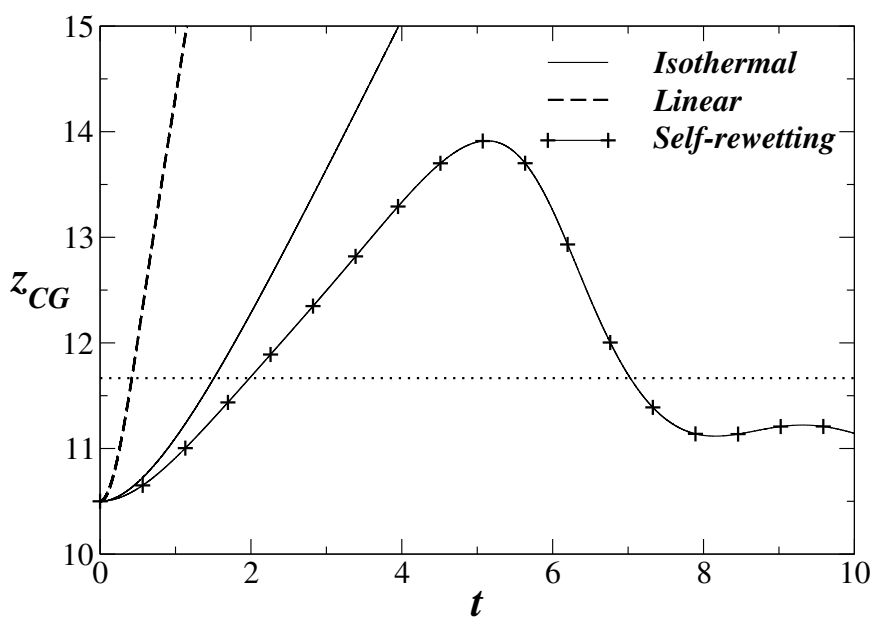

Figure 4: Temporal variation of the center of gravity of the bubble for the parameter values $\mathrm{Ga}=10, B o=10^{-2}, \rho_{r}=10^{-3}, \mu_{r}=10^{-2}, \Gamma=0.1$ and $\alpha_{r}=0.04$. The plots for the isothermal $\left(M_{1}=0\right.$ and $\left.M_{2}=0\right)$, linear $\left(M_{1}=0.4\right.$ and $\left.M_{2}=0\right)$ and self-rewetting $\left(M_{1}=0.4\right.$ and $\left.M_{2}=0.2\right)$ cases are shown in the figure. The horizontal dotted line indicates the prediction of Eq. (4.1) for the self-rewetting case.

seen from this figure that following an initial, relatively short, acceleration period, the bubble reaches a constant, terminal speed for both the isothermal case, and the linear fluid in the non-isothermal case. The terminal velocity is higher for the non-isothermal case due to the presence of Marangoni stresses driving liquid towards the cold region of the tubel and thereby enhancing the upward motion of the bubble. For the self-rewetting fluid in the non-isothermal case, $z_{C G}$ also reaches a constant speed for a certain time duration; this is, however, followed by a drop in $z_{C G}$ before a terminal $z_{C G}$ value is reached. Thus, the motion of a bubble rising initially in a self-rewetting fluid, whose temperature is essentially increasing linearly, is first reversed and then arrested. The fact that the bubble motion comes to a halt in the self-rewetting and not the linear fluid in a positive temperature gradient was also suggested by Eq. (3.36) in the Stokes flow limit. This property can be used to manipulate bubbles by simply shifting the temperature gradient along the wall appropriately. This might be of interest to researchers working in microfluidics and multiphase microreactors. The predictions of the dimensionless version of Eq. (3.41), given by

$$
z_{c}=\left(\frac{\Gamma M_{1}}{2}+\frac{B o}{3}\right) \frac{1}{M_{2} \Gamma^{2}},
$$

are also shown in Fig. 4. For the parameters used to generate the results presented in this figure, $z_{c} \sim 11.67$, which is in good agreement with the numerical predictions, despite the fact that strong inertial contributions are present in the flow as represented by $G a=10$. We will examine the mechanism underlying this behaviour below.

In Fig. 5a, we examine the dependence of the terminal velocity $V_{t}$ of a bubble rising in a linear fluid on the parameter $M_{1}$; the latter governs the strength of the linear variation of the surface tension with temperature. As explained above, for positive values of $\Gamma$ the induced Marangoni stresses increase the rise velocity of the bubble. The terminal velocity, though, appears to reach a plateau with $M_{1}$ indicating that the strength of Marangoni stresses saturates at large $M_{1}$. For a spherical bubble rising in an unconfined fluid, according to the analytical solution Eq. (3.30) the terminal velocity is predicted 
(a)

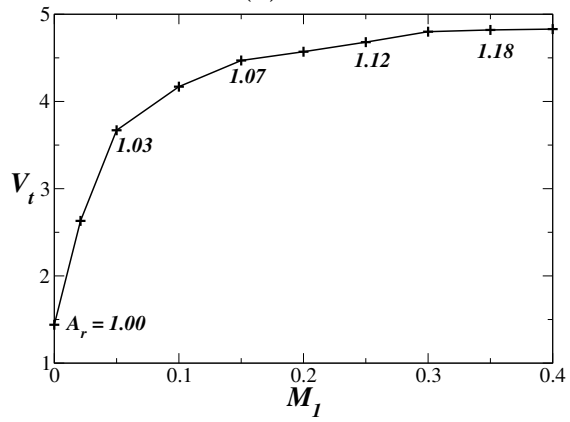

(b)

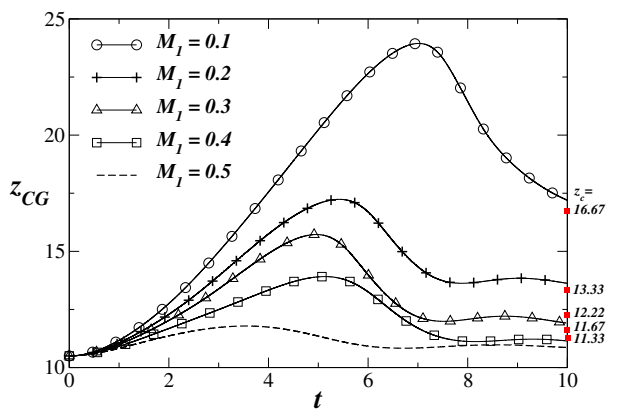

(c)

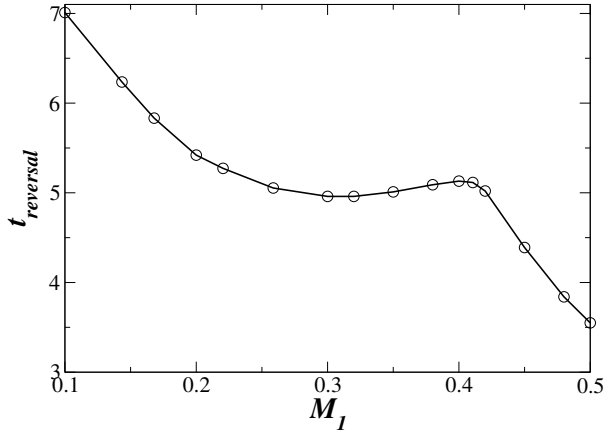

Figure 5: (a) The terminal velocity of the center of gravity of the bubble for different values of $M_{1}$ for $M_{2}=0$; (b) temporal variation of the center of gravity of the bubble for $M_{2}=M_{1} / 2 ;$ (c) variation of the time at which $z_{C G}$ reaches its maximum for different values of $M_{1}$. The rest of the parameter values are $\mathrm{Ga}=10, B o=10^{-2}, \rho_{r}=10^{-3}$, $\mu_{r}=10^{-2}, \Gamma=0.1$ and $\alpha_{r}=0.04$. The inserted numbers in panel (a) are the values of aspect ratio, $A_{r}(\equiv w / h)$ of the bubble for different values of $M_{1}$, wherein $h$ and $w$ represent the height and width of the bubble, respectively. The dotted line represents the analytical result for unbounded domain in the Stokes flow limit obtained from Eq. (3.30) . The numerical predictions of Eq. (4.1) are shown by the filled square symbols on the right vertical axis of panel (b).

to be proportional to the parameter $M_{1}$. However in this case we consider a deformable bubble which has a important effect on the flow. As the bubble rises, the interfacial tension continuously decreases since the bubble experiences higher temperatures. Although the $B o$ evaluated at the reference temperature is rather small indicating a relatively undeformable bubble, locally the $B o$ increases allowing the bubble to deform significantly. As it is indicated in Fig. 5a the aspect ratio $A_{r}(\equiv w / h)$ of the bubble increases (becomes more oblate) with increasing $M_{1}$, which in turn increases the drag (Mahesri et al. 2014) resisting to the upward motion of the bubble.

In the case of self-rewetting fluids, one parameter that we need to take into account is the position of minimum surface tension with respect to the bubble because it will affect the action of induced Marangoni stresses; this effect will be studied in detail below. For the time being, we have positioned the center of gravity of the bubble above the position of minimum surface tension $\left(z_{i}=10.5, z_{m}=10\right)$ at $t=0$. In this case, as the bubble rises, it comes into contact with liquid of increasingly lower surface tension. The induced Marangoni stresses drive liquid upwards, towards the hot region of the tube, and inhibit 


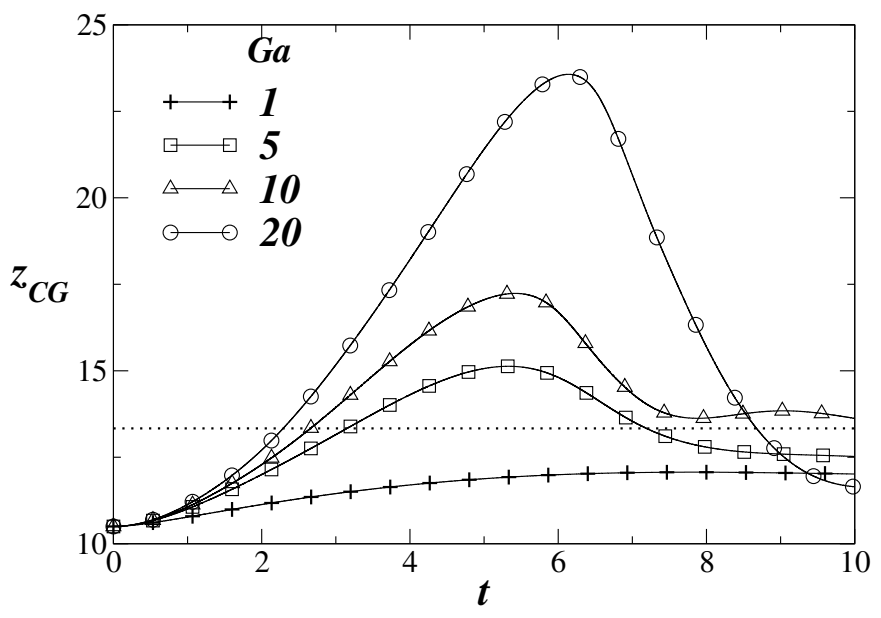

Figure 6: Effect of $\mathrm{Ga}$ on the temporal evolution of the bubble centre of gravity for $B o=10^{-2}, \rho_{r}=10^{-3}, \mu_{r}=10^{-2}, M_{1}=0.2, M_{2}=0.1, \Gamma=0.1$ and $\alpha_{r}=0.04$. The prediction of Eq. (4.1) is shown by the dotted line.

the upward motion of the bubble. This is evident at early times in Fig. 4 where it is shown that the rise velocity for the self-rewetting fluid is lower than for the isothermal case.

In order to study the effect of Marangoni stress in more detail, we examine in Fig. 5b the terminal distance reached by bubbles moving in a self-rewetting fluid as a function of $M_{1}$ with $M_{2}=M_{1} / 2$; the latter restriction is imposed in order to keep the position where the minimum surface tension arises constant. As shown in Fig. 5b, this distance increases with decreasing $M_{2}$, which indicates that an increase in the self-rewetting character of the fluid leads to a larger degree of bubble retardation: in the limit $M_{2} \rightarrow 0$, a steady, terminal speed is reached for $\Gamma>0$. The numerical predictions for the terminal distance shown in Fig. 5b are also in good agreement with those obtained from Eq. (4.1): for the parameters used here, $z_{c} \sim(16.67,13.33,12.22,11.67,11.33)$ for $M_{1}=(0.1,0.2,0.3,0.4,0.5)$. Also, for $B o \sim 0$, and $M_{2}=M_{1} / 2$, Eq. (4.1) reduces to $z_{c} \sim \Gamma^{-1}$, which for the parameters in Fig. $5 \mathrm{~b}$ leads to $z_{c} \sim 10$; this appears to be the value to which the terminal distance limits with increasing $M_{1}$.

Interestingly, the onset time for motion reversal, $t_{\text {reversal }}$, has a non-monotonic dependence on $M_{1}$ : starting from a global maximum at small $M_{1}, t_{\text {reversal }}$ exhibits a shallow minimum, followed by a local maximum, before undergoing a sharp decrease with increasing $M_{1}$. This is probably due to the effect of inertia and the interplay of buoyancy and Marangoni stresses which act in opposite directions. For low $M_{1}$ values, the Marangoni stresses are initially relatively weak and take a long time before they grow to change the direction of motion of the bubble. As $M_{1}$ increases, Marangoni stresses become stronger initially, so the bubble decelerates faster and less time is needed for the motion reversal. However, as Marangoni stresses gain in relative significance, the initial acceleration of the bubble becomes considerably smaller and this results in small rise velocities initially and therefore the bubble now has to move for longer times before it reaches the position of motion reversal. Finally for even higher values of $M_{1}$, the Marangoni stresses are so strong that they outweigh buoyancy, and the bubble very soon starts moving in the opposite direction.

The effect of inertia on the bubble motion in a self-rewetting fluid, parameterised by 
the Galileo number, $G a$, is also of interest, and is shown in Fig. 6. As can be seen from this figure, at low values of $G a$, the bubble centre of gravity, $z_{C G}$, increases monotonically with time before reaching a terminal value. Increasing the $G a$ number leads to increase of the initial rise velocity of the bubble, probably due to the fact that higher values of $G a$ correspond to fluids of lower viscosity. The most interesting effect, however, is that the bubble exhibits flow reversal; the maximal $z_{C G}$ values reached increase progressively with $G a$ prior to flow reversal, which then culminates in the bubble motion being arrested. The onset of flow reversal also appears to be an increasing function of $\mathrm{Ga}$. For the parameters used in Fig. 6, we find using Eq. (4.1) that $z_{c} \sim 13.33$. As it is shown in Fig. 6 this value is quite close to our calculations for the terminal position of the bubble even for high values of $G a$ and in the presence wall confinement despite the fact that Eq. (4.1) was derived an unconfined bubble moving in the Stokes flow limit. This is explained by the fact that at the latter stages of the flow, for all values of $G a$, the migration velocity of the bubble decreases significantly, entering into the creeping flow regime.

Next we examine the effect of mean surface tension, characterised by the Bond number, $B o$, on the bubble dynamics in a self-rewetting fluid; this is shown in Fig. 7a,b for $G a=5$ and $G a=10$, respectively. It is seen clearly in Fig. 7a,b that there exists a critical value of $B o$ above which flow reversal is no longer possible and $z_{C G}$ undergoes a monotonic rise with time whose rate decreases, and eventually saturates, with increasing $B o$. These results highlight the role of bubble deformation in the dynamics: minimising deformation, which is promoted by small values of $B o$, accelerates flow reversal, leading to lower terminal $z_{C G}$ values. For $G a=5$, measures of interfacial deformation are provided by the bubble length, $l_{B}$ and aspect ratio of the bubble, $A_{r}$ whose temporal variation are shown in Fig. 7c,d and Fig. 7e,f, respectively for small and large $B o$ values. Inspection of these panels reveals that the extent of deformation increases with $B o$, as expected.

In order to elucidate the reasons underlying the behaviour depicted in Fig. 7, we show in Fig. 8 the evolution of the bubble shape and that of the temperature distribution in the fluid surrounding the bubble for two values of $B o$; the rest of the parameters remain fixed at their 'base' values. Also shown in Fig. 8 are streamlines which represent the structure of the flow within the bubble, in the surrounding fluid flowing past it, as well as in its wake region. It is seen that the bubble in the $B o=10$ case (shown in Fig. 8a), which rises starting from $z_{i}=10$ that coincides with the surface tension minimum in Fig. 2, undergoes significant deformation; this begins at relatively early times, and culminates in the formation of a cap bubble. This deformation is accompanied by the formation of a pair of counter-rotating vortices within the bubble, and another pair in the wake region; the lateral and vertical extent of the latter increase with time, as the bubble rises towards the warmer regions of the tube. No evidence of flow reversal is observed, which is consistent with the results presented in Fig. 7.

For the $B o=10^{-2}$ case, it is seen from Fig. $8 \mathrm{~b}$ that the bubble suffers negligible deformation, and its rise is accompanied by the formation of a pair of counter-rotating vortices form inside the bubble at early times, as was also observed in the $B o=10$ case (shown in Fig. 8a). This flow structure persists until $t=5$ at which the bubble is seen to develop a wake region, and two more vortices are formed within the bubble; this coincides with the onset of flow reversal, as can be ascertained upon inspection of Fig. 7a. At later times, the direction of the flow is reversed as indicated by the direction of the streamlines associated with the $t=10$ panel for $B o=10^{-2}$ in Fig. 8b, which points upwards since the liquid flows past a descending bubble. This is brought about by the fact that the vertical temperature gradient across the bubble is positive which gives rise to a positive surface tension gradient since $z>z_{m}$ (viz. Fig. 2). This, then, sets up a Marangoni stress, which acts in the opposite direction to the flow past the rising bubble, 
(a)

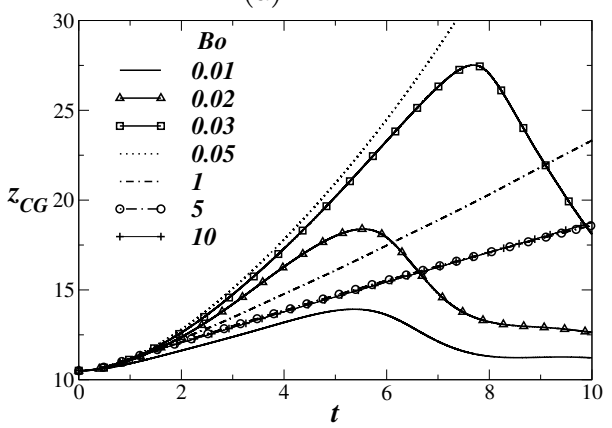

(c)

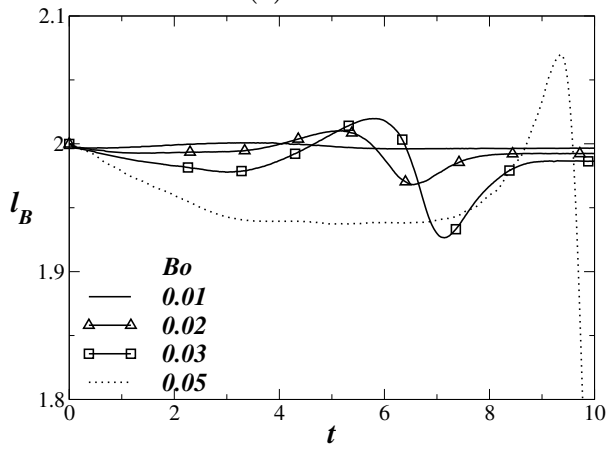

(e)

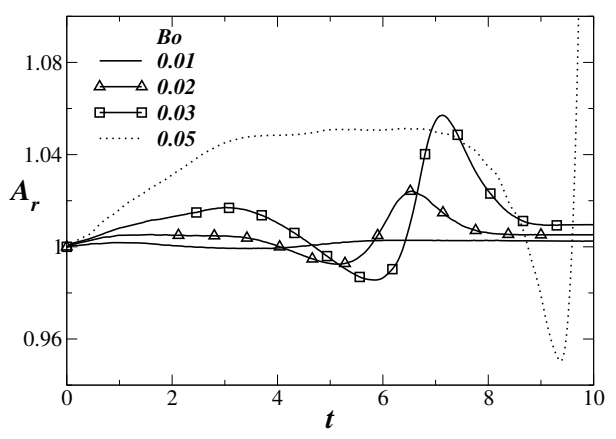

(b)

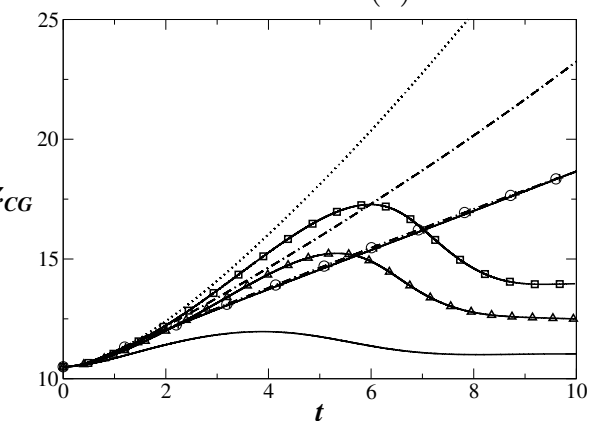

(d)

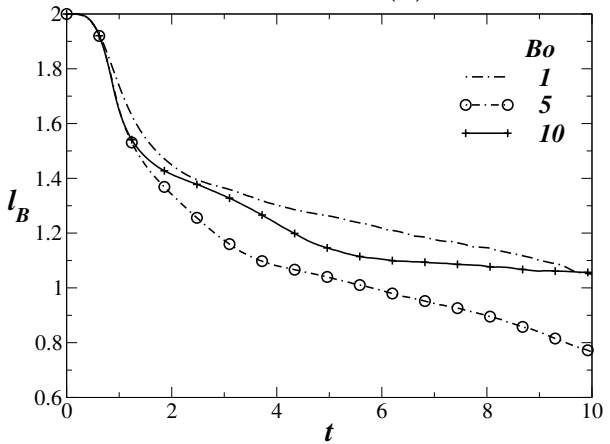

(f)

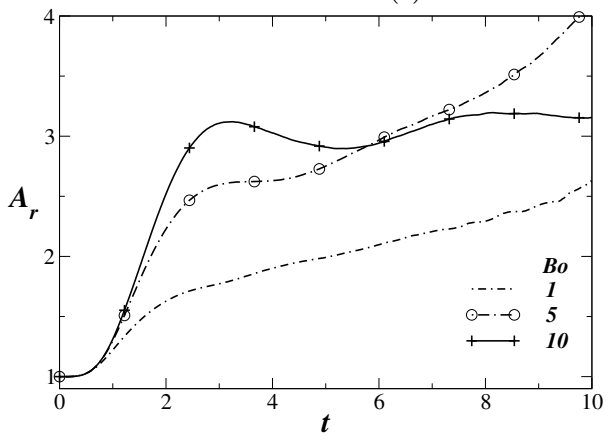

Figure 7: Effect of $B o$ on bubble motion for (a) $G a=10$ and (b) $G a=5$; effect of $B o$ on the $(\mathrm{c}, \mathrm{d})$ length of the bubble, $l_{B}$, (e,f) aspect ratio of the bubble, $A_{r}$ for $G a=5$. The rest of the parameters values $\rho_{r}=10^{-3}, \mu_{r}=10^{-2}, M_{1}=0.4, M_{2}=0.2, \Gamma=0.1$ and $\alpha_{r}=0.04$.

retarding its motion. This stress becomes increasingly dominant, counterbalances, and then exceeds the magnitude of the buoyancy force, leading to the reversal of the bubble motion and its eventual arrest.

We have also studied the effect of varying the initial location of the bubble on the dynamics of the centre of gravity of bubble, $z_{C G}$. In Fig. 9, we show the temporal evolution of $z_{C G}$ as a parametric function of $z_{i}$ with the rest of the parameters fixed at their 'base' values. For situations in which the initial location of the bubble is lower than that associated with the surface tension minimum in Fig. 2, $z=z_{m}$, the surface tension gradient across the bubble re-inforces the buoyancy-driven bubble rise. This results in an increase in $z_{C G}$ with time until the bubble reaches elevations such that $z>z_{m}$ for 
(a)

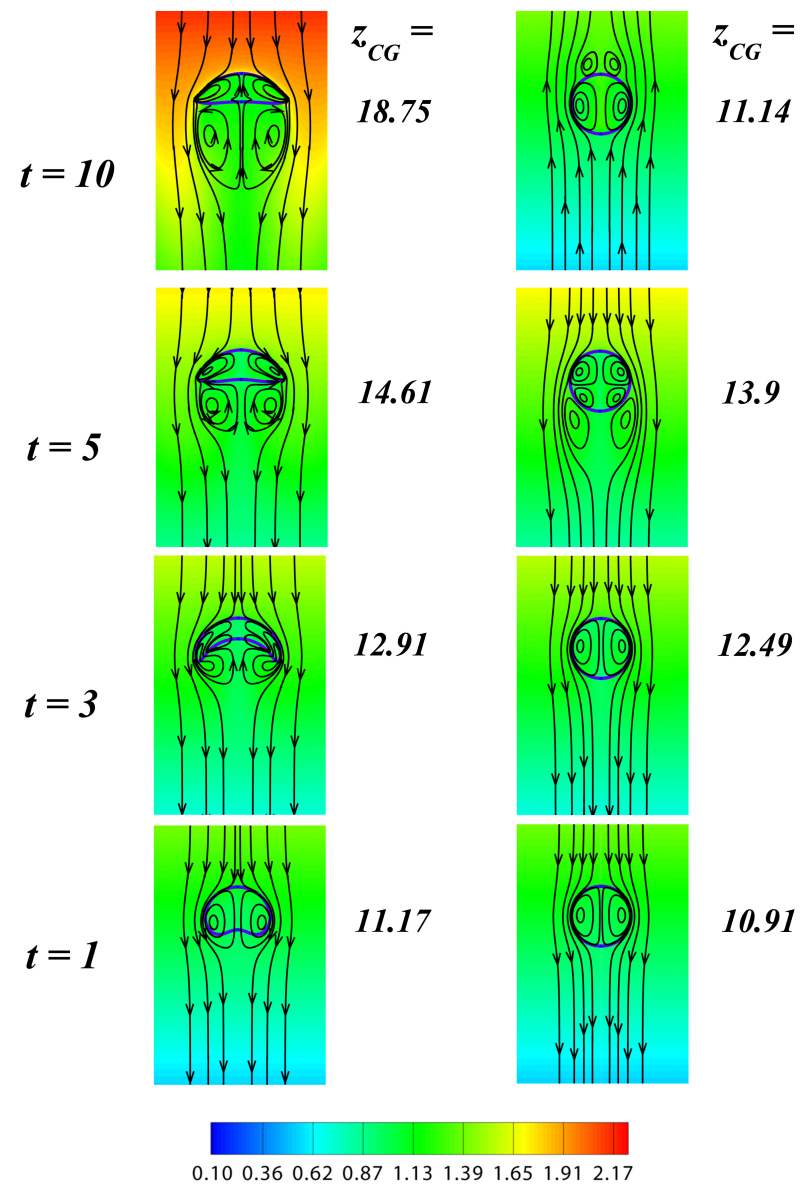

Figure 8: Evolution of bubble shape (blue line), streamlines (lines with arrows), and temperature contours (shown in color) with time for (a) $B o=10$ and (b) $B o=10^{-2}$. The initial location of the bubble, $z_{i}=10$. The inset at the bottom represents the colormap for the temperature contours. The rest of the parameter values are $\mathrm{Ga}=10$, $\rho_{r}=10^{-3}, \mu_{r}=10^{-2}, M_{1}=0.4, M_{2}=0.2, \Gamma=0.1$ and $\alpha_{r}=0.04$.

which the sign of the surface tension gradient across the bubble is reversed, which drives Marangoni flow that acts to retard and eventually reverse the direction of bubble motion. The time associated with the onset of flow reversal decreases with increasing $z_{i}$. For sufficiently large values of $z_{i}$, the bubble moves in the negative $z$-direction under the action of Marangoni stresses whose magnitude exceeds that of the buoyancy force. The terminal value of $z_{C G}$ appears to be weakly-dependent on $z_{i}$ for large $z_{i}$ values. Also, $z_{c} \sim 13.33$ from Eq. (4.1), which is in good agreement with the numerical predictions.

As was mentioned in the introduction, self-rewetting fluids have been used in heat pipes associated with substantially higher heat fluxes than normal liquids. In these applications, the bubbles are very confined, usually forming slugs. It therefore seems appropriate to investigate the effect of confinement. We have done this by varying the value of the dimensionless radius of the tube, $H$ and plotted in Fig. 10a the temporal evolution of the bubble length, $l_{B}$, for $B o=10$ and $B o=100$, and $H=2.5$. For this set of simulations 


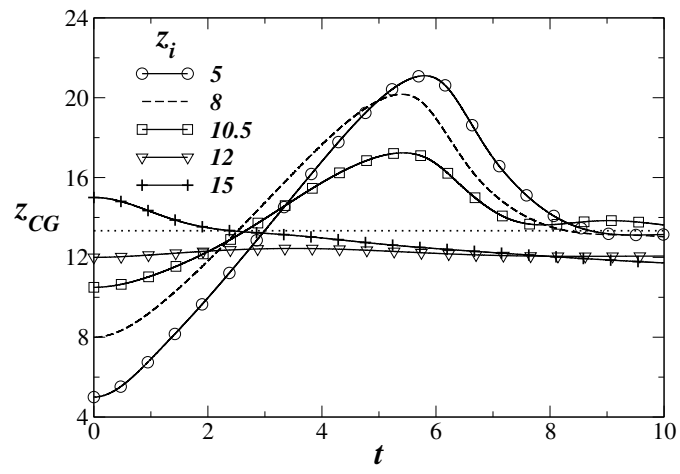

Figure 9: The effect of initial location of the bubble on the temporal evolution of the center of gravity, $z_{C G}$. The rest of the parameter values are $\mathrm{Ga}=10, B o=10^{-2}$, $\rho_{r}=10^{-3}, \mu_{r}=10^{-2}, M_{1}=0.2, M_{2}=0.1, \Gamma=0.1$ and $\alpha_{r}=0.04$. The prediction of Eq. (4.1) is shown by the dotted line.

(a)

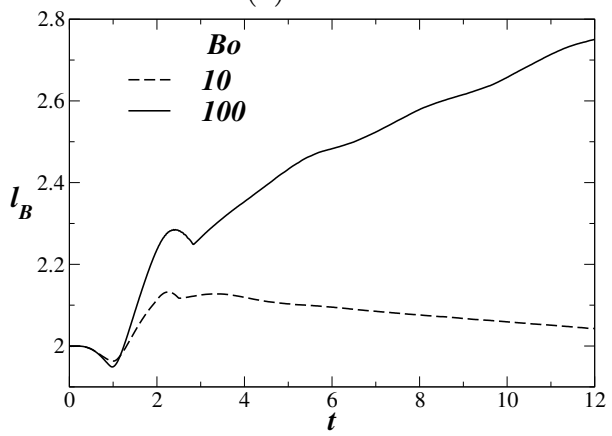

(b)

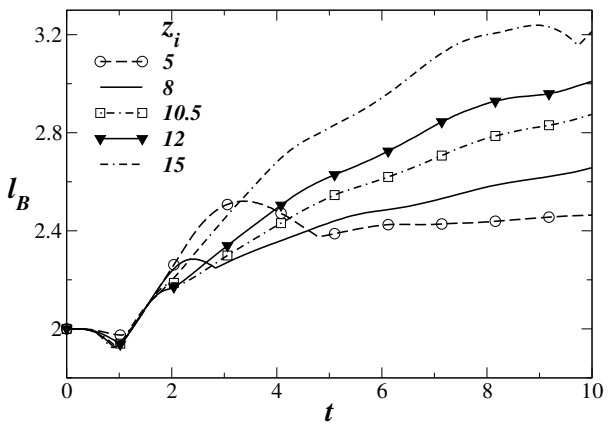

Figure 10: (a) Evolution of the length of the bubble, $l_{B}$ for two values of $B o$ when he initial location of the bubble $z_{i}=8$. (b) The effects of initial location of the bubble on elongation of the bubble for $B o=100$. The radius of the tube, $H=2.5$. The rest of the parameters are $\mathrm{Ga}=10, \rho_{r}=10^{-3}, \mu_{r}=10^{-2}, M_{1}=0.4, M_{2}=0.2, \Gamma=0.1$ and $\alpha_{r}=0.04$.

we place the bubble below the position of minimum surface tension $\left(z_{i}=8, z_{m}=10\right)$. As seen in this figure, the bubble undergoes a contraction at early times, which is followed by rapid expansion for both values of $B o$. This is then followed by a sustained increase (decrease) in $l_{B}$ with time for $B o=100(B o=10)$. The effect of the initial location of the bubble, $z_{i}$ on the elongation of the bubble is investigated in Fig. 10b. It can be seen that the length of the bubble, $l_{B}$ increases as we move the initial location of the bubble in the positive $z$ direction.

The evolution of the bubble shape, temperature distribution, and flow structure for the dynamics associated with $B o=10$ and $B o=100$ for the parameter values the same as those used in Fig. 10a are shown in Fig. 11. Inspection of this figure reveals that the bubble remains essentially bullet-shaped for $B o=10$, which is in contrast to the caplike shape adopted by the bubble for the same $B o$ and larger $H$ value. For $B o=100$, the bubble develops filaments in its wake region, driven by the formation of a pair of counter-rotating vortices in this region, which leads to bubble elongation. This elongation 
(a)

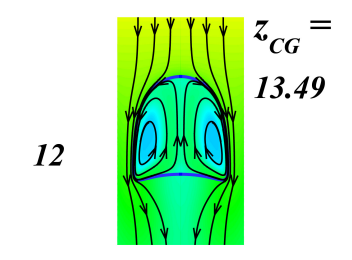

8
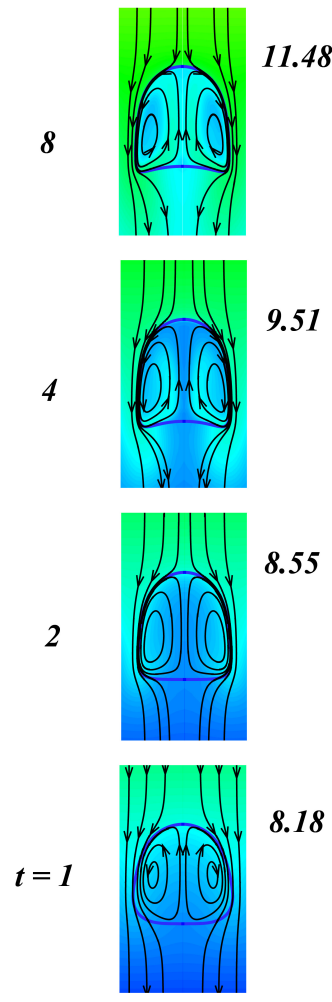

(b)
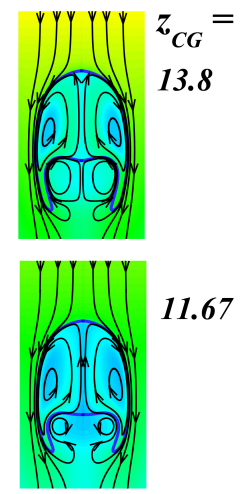

11.67

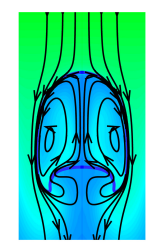

9.59

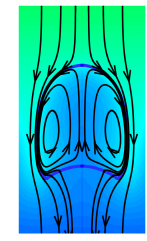

8.58

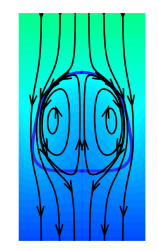

8.18

Figure 11: Evolution of bubble shape (blue line), streamlines (lines with arrows), and temperature contours (shown in color) with time for (a) $B o=10$ and (b) $B o=100$, and $H=2.5$. The initial location of the bubble $z_{i}=8$. The inset at the bottom represents the colormap for the temperature contours. The rest of the parameters are $\mathrm{Ga}=10$, $\rho_{r}=10^{-3}, \mu_{r}=10^{-2}, M_{1}=0.4, M_{2}=0.2, \Gamma=0.1$ and $\alpha_{r}=0.04$.

is sustained by the action of the vortices whose size grows with time and they cause the stretching of the filaments from the main body of the bubble towards the wake region.

Next, we study the effect of self-rewetting character of the liquid surrounding the bubble on its elongation in the presence of confinement effects. The results are shown in Fig. 12 for $B o=10, G a=5$ while the tube radius has been reduced to $H=2.1$ to intensify the effect of confinement; the rest of the parameters remain fixed at their 'base' values. It is seen clearly from Fig. 12a that the bubble elongation rate increases with $M_{2}\left(=M_{1} / 2\right)$; the maximal $l_{B}$ is reached at an earlier time with increasing $M_{2}$. For the largest $M_{2}$ values studied, the bubble undergoes a weak contraction to an essentially 
(a)

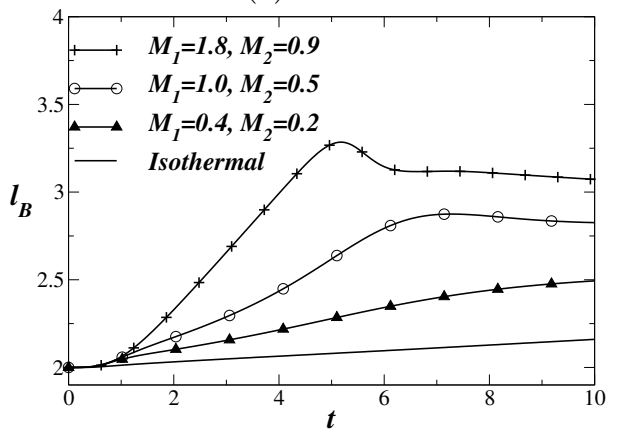

(b)

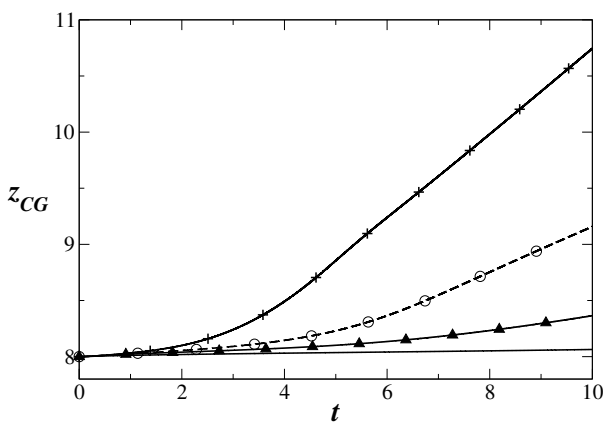

Figure 12: Evolution of (a) the length of the bubble, $l_{B}$, (b) the location of center of gravity, in a tube having $H=2.1$. The initial location of the bubble $z_{i}=8$. The rest of the parameters are $\mathrm{Ga}=5, \rho_{r}=10^{-3}, \mu_{r}=10^{-2}$. The non-isothermal curve is plotted for $\Gamma=0.1$ and $\alpha_{r}=0.04$.

terminal $l_{B}$ value. The bubble rise speed also increases with $M_{2}$, as shown in Fig. 12b which depicts the temporal evolution of the bubble centre of gravity, $z_{C G}$.

We contrast in Fig. 13 the flow dynamics associated with the isothermal and $\left(M_{1}=\right.$ 1.8, $M_{2}=0.9$ ) cases shown in Fig. 12. It is seen that in contrast to the isothermal case in which the bubble shape remains approximately spherical, the bubble rising in a nonisothermal, self-rewetting fluid deforms significantly in the presence of confinement, and assumes the shape of a Taylor bubble. It is difficult to compare this with the behaviour of a bubble in a linear fluid since there is no obvious basis for such a comparison.

\section{Concluding remarks}

We have carried out an analytical and numerical investigation of a gas bubble rising in a non-isothermal liquid in a cylindrical tube whose walls have a linearly-increasing temperature. Two types of liquids were considered: a 'linear' liquid whose surface tension decreases linearly with temperature; and a so-called 'self-rewetting' liquid which exhibits a parabolic dependence of the surface tension on temperature, with a welldefined minimum. Attention was focused on how the latter can affect the development of thermocapillary Marangoni stresses and, in turn, the bubble dynamics.

We have shown that in the Stokes flow limit, the motion of a spherical bubble can be arrested in a self-rewetting liquid, and derived a formula for the terminal distance in this case, even if the temperature gradient in this liquid, which surrounds the bubble, is positive. This is in contrast to the case of a linear liquid in which a negative gradient is necessary to bring the bubble motion to a halt. We have also studied the bubble motion numerically to account for the presence of thermocapillarity, buoyancy, inertia, interfacial deformation, and confinement effects. Our results have demonstrated that the motion of the bubble in a self-rewetting fluid can be reversed and then arrested in the limit of weak bubble deformation. In this limit, good agreement between the numerical and analytical predictions for the terminal distance was found, even for appreciable inertial contributions; this is due to the fact that during the latter stages of the flow, the bubble enters the creeping flow regime prior to reaching its terminal location. The flow reversal becomes accentuated for strongly self-rewetting liquids in the presence of significant inertia. These phenomena are absent in the case of linear liquids and are attributed to the thermocapillary Marangoni stresses which oppose the direction of the buoyancy-driven 
(a)

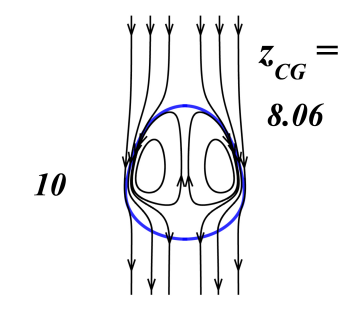

6

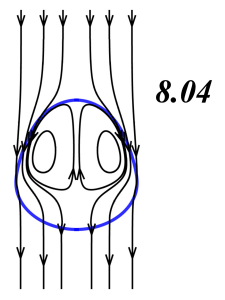

2
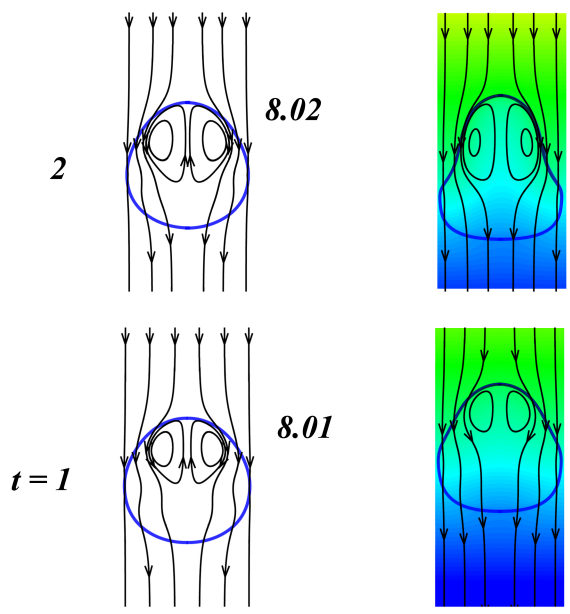

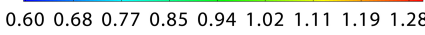

(b)

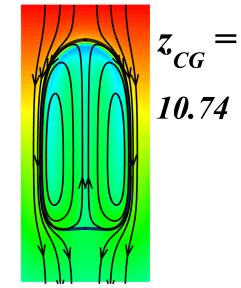

9.24

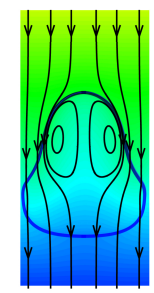

8.1

8.03

Figure 13: Evolution of bubble shape with time for (a) isothermal case, and (b) $M_{1}=$ $1.8, M_{2}=0.9$ (temperature contours shown in color). The inset at the bottom represents the colormap for the temperature contours. The rest of the parameters are the same as those used to generate Fig. 12.

bubble rise when the bubble crosses the vertical location associated with the surface tension minimum. These stresses gain in significance during the course of the flow and eventually become dominant leading to reversal and arrest of the bubble motion.

We have also shown that a bubble in a self-rewetting fluid undergoes considerable elongation for significant confinement, forming a Taylor bubble; this is absent in the case of isothermal flows in which the bubble remains essentially spherical.

OKM wishes to thank the Engineering and Physical Sciences Research Council, UK, for their support through the MEMPHIS Programme Grant (number EP/K003976/1). KCS would also like to thank Indian National Science Academy for the financial support. 
We thank the anonymous referee for providing an alternative derivation (included in the appendix) of creeping flow calculation given in section 3 .

\section{Appendix}

If we assume that convective heat transfer and drop deformation are negligible., the governing equations are linear and the motion due to gravity and thermocapillarity can be treated separately. Thus we write the drift velocity of the bubble as $U=U_{g}+U_{t}$, where the gravitational contribution

$$
U_{g}=-\frac{\rho R^{2}}{3 \mu} g .
$$

The thermocapillary contribution $U_{t}$ can be determined by solving the creeping flow problem.

$$
\begin{gathered}
\mu \nabla^{2} \mathbf{u}=\nabla p \quad \text { and } \quad \nabla \cdot \mathbf{u}=0, \\
n \cdot \tau \cdot(I-n n)=-\nabla_{s} \sigma \quad \text { at } \quad \mathrm{r}=\mathrm{R}, \\
n \cdot \mathbf{u}=0 \quad \text { at } \quad \mathrm{r}=\mathrm{R}, \\
\mathbf{u} \rightarrow-U_{t} \mathbf{e}_{z} \quad \text { as } \quad \mathrm{r} \rightarrow \infty,
\end{gathered}
$$

where $\tau=-P+\left(\nabla \mathbf{u}+\nabla \mathbf{u}^{T}\right) / 2, \nabla_{s}$ is the surface gradient operator, $n$ is the outward normal vector to the bubble surface. Additionally, the drop is force-free in the thermocapillary problem, hence $\int_{S} \tau \cdot n \mathrm{~d} S=0$, where the integral is over the bubble surface $S$. Now, we invoke the Lorentz reciprocal theorem (exterior to the bubble), which states

$$
\int_{A} \mathbf{v} \cdot \tau \cdot n \mathrm{dA}+\int_{\mathrm{S}} \mathbf{v} \cdot \tau \cdot \mathrm{ndS}=\int_{\mathrm{A}} \mathbf{u} \cdot \Sigma \cdot \mathrm{ndA}+\int_{\mathrm{S}} \mathbf{u} \cdot \Sigma \cdot \mathrm{ndS}
$$

where $A$ denotes an integral over a surface at infinity. In (6.6), $\mathbf{v}$ and $\Sigma$ are the velocity and stress fields belonging to a companion Stokes flow, which we take to be that produced by a bubble at rest in a uniform stream $\mathbf{V}$. The first integral on the left of $(6.6), \int_{A} \mathbf{v} \cdot \tau \cdot n \mathrm{dA}=$ $-\mathbf{V} \cdot \int_{\mathrm{A}} \tau \cdot \mathrm{ndA}=0$ since the bubble is force free in the thermocapillary problem. The first integral on the right, $\int_{A} \mathbf{u} \cdot \Sigma \cdot n \mathrm{dA}=-\mathrm{U}_{\mathrm{t}} \mathbf{e}_{\mathrm{z}} \cdot \int_{\mathrm{A}} \Sigma \cdot \mathrm{ndA}=-4 \pi \mu \mathrm{RV} \cdot\left(\mathrm{U}_{\mathrm{t}} \mathbf{e}_{\mathrm{z}}\right)$, in which we have used the well known result for the drag on a bubble. For the second integral on the left, note that $\mathbf{v}=\frac{1}{2} \mathbf{V} \cdot(I-n n)$ is the (purely tangential) fluid velocity at the bubble surface; hence, using the tangential stress balance in the thermocapillary problem gives $\int_{S} \mathbf{v} \cdot \tau \cdot n \mathrm{dS}=\frac{1}{2} \mathbf{V} \cdot \int_{\mathrm{S}} \nabla_{\mathrm{S}} \sigma \mathrm{dS}$. Last, the second integral on the right of (6.6) equals zero as there is no shear stress on the bubble in the complimentary problem. Thus, we arrive at the following expression for the bubble velocity in the thermocapillary problem

$$
U_{t} \mathbf{e}_{z}=-\frac{1}{8 \pi \mu R} \int_{S} \nabla_{s} \sigma \mathrm{dS}
$$

which is essentially an average of the Marangoni stress over the bubble. Notably, (6.7)

obviates the need to find the detailed flow field and is valid for any relation of surface tension to temperature.

For a self-rewetting fluid the surface tension is given by eq. (2.5) We assume the bubble is a thermal insulator; hence, the temperature profile in the liquid

$$
T=T_{1}+\mathbf{h} \cdot \mathbf{r}_{b}+\left(1+\frac{R^{3}}{2 r^{3}}\right) \mathbf{h} \cdot \mathbf{r},
$$

where $\mathbf{h}$ is the constant temperature gradient vector, $\mathbf{r}_{b}$ is the position of the bubble 
center, and $\mathbf{r}$ is the position vector from the center. The difference between the temperature at the bubble center and the reference temperature $T_{1}$ is equal to $\mathbf{h} \cdot \mathbf{r}_{b}$, which is equivalent to the quantity $T_{\infty}(0)-T_{1}$ in section 3 . Inserting (6.8) into (2.5) yields

$$
\sigma=\sigma_{0}+\left(-\beta_{1}+2 \beta_{2} \mathbf{h} \cdot \mathbf{r}_{b}\right)\left(1+\frac{R^{3}}{2 r^{3}}\right) \mathbf{h} \cdot \mathbf{r}+\beta_{2}\left(\mathbf{h} \cdot \mathbf{r}_{b}\right)^{2}+\beta_{2}\left(1+\frac{R^{3}}{2 r^{3}}\right)^{2}(\mathbf{h} \cdot \mathbf{r})^{2} .
$$

The first and third terms in the above are constant and therefore do not generate a Marangoni stress. The fourth term generates a Marangoni stress that is symmetric about the bubble centre, which is due to the quadratic dependence of the surface tension on $\beta_{2}$. This symmetry dictates that the fourth term does not yield a contribution to the thermocapillary velocity of the bubble. However, the second term does drive a Marangoni stress that contributes to the bubble velocity; specifically, from (6.9)

$$
\nabla_{s} \sigma=\frac{3}{2}\left(-\beta_{1}+2 \beta_{2} \mathbf{h} \cdot \mathbf{r}_{b}\right) \mathbf{h} \cdot(I-n n)+\cdots,
$$

where $\cdots$ indicate the Marangoni stress from the fourth term in (6.9) that, as mentioned above, does not contribute to the bubble velocity. Inserting (6.10) into (6.7) and performing the required integration yields

$$
U_{t}=\frac{\beta_{1} R}{2 \mu}\left(1-\frac{2 \beta_{2}}{\beta_{1}} \mathbf{h} \cdot \mathbf{r}_{b}\right),
$$

which when summed with the gravitational velocity (6.1) yields the total bubble velocity given in equation (3.36). This derivation is provided by the anonymous referee.

\section{REFERENCES}

Abe, Y., Iwasaki, A. \& TANaka, K. 2004 Microgravity experiments on phase change of selfrewetting fluids. Ann. N.Y. Acad. Sci. 1027, 269285.

Acrivos, A., Jeffrey, D. J. \& Saville, D. A. 1990 Particle migration in suspensions by thermocapillary or electrophoretic motion. Journal of Fluid Mechanics 212, 95110.

Balasubramaniam, R. 1998 Thermocapillary and buoyant bubble motion with variable viscosity. International Journal of Multiphase Flow 24 (4), 679-683.

Balasubramaniam, R \& Chai, An-Ti 1987 Thermocapillary migration of droplets: An exact solution for small marangoni numbers. Journal of Colloid and Interface Science 119 (2), 531-538.

Balasubramaniam, R. \& Subramaniam, R. S. 1996 Thermocapillary bubble migrationthermal boundary layers for large marangoni numbers. International Journal of Multiphase Flow 22 (3), 593-612.

Balasubramaniam, R. \& Subramanian, R. Shankar 2000 The migration of a drop in a uniform temperature gradient at large marangoni numbers. Physics of Fluids (1994-present) 12 (4), 733-743.

Balasubramaniam, R. \& Subramanian, R. Shankar 2004 Thermocapillary convection due to a stationary bubble. Physics of Fluids (1994-present) 16 (8), 3131-3137.

Borcia, Rodica \& Bestehorn, Michael 2007 Phase-field simulations for drops and bubbles. Physical Review E $\mathbf{7 5}$ (5), 056309.

Brackbill, JU, Kothe, Douglas B \& Zemach, C1 1992 A continuum method for modeling surface tension. J. Comput. Phys 100, 335-354.

Brady, P. T., Herrmann, M. \& Lopez, J. M. 2011 Confined thermocapillary motion of a three-dimensional deformable drop. Physics of Fluids (1994-present) 23 (2), 022101.

Chen, Jinnan, Dagan, Zeev \& Maldarelli, Charles 1991 The axisymmetric thermocapillary motion of a fluid particle in a tube. Journal of Fluid Mechanics 233, 405437.

Chen, J. C. \& Lee, Y. T. 1992 Effect of surface deformation on thermocapillary bubble migration. AIAA Journal 30 (4), 993-998. 
Crespo, A., Migoya, E. \& Manuel, F. 1998 Thermocapillary migration of bubbles at large reynolds numbers. International Journal of Multiphase Flow 24 (4), 685-692.

Ding, H., Spelt, P. D. M. \& Shu, C. 2007 Diffuse interface model for incompressible two-phase flows with large density ratios. J. Comput. Phys 226, 2078-2095.

Haj-Hariri, H., Shi, Q. \& Borhan, A. 1997 Thermocapillary motion of deformable drops at finite reynolds and marangoni numbers. Physics of Fluids (1994-present) 9 (4), 845-855.

Hasan, M. \& Balasubramaniam, R. 1989 Thermocapillary migration of a large gas slug in a tube. Journal of Thermophysics and Heat Transfer 3 (1), 87-89.

Herrmann, M., Lopez, J. M., Brady, P. \& Raessi, M. 2008 Thermocapillary motion of deformable drops and bubbles. In Proceedings of the Summer Program 2008, p. 155. Stanford University: Center for Turbulence Research.

Hu, Y., Liu, T., LI, X. \& WANG, S. 2014 Heat transfer enhancement of micro oscillating heat pipes with self-rewetting fluid. Int. J. Heat Mass Trans. 70, 496-503.

Karapetsas, G., Sahu, K. C., Sefiane, K. \& Matar, O. K. 2014 Thermocapillary-driven motion of a sessile drop: effect of non-monotonic dependence of surface tension on temperature. submitted for publication .

Keh, Huan J., Chen, Po Y. \& Chen, Li S. 2002 Thermocapillary motion of a fluid droplet parallel to two plane walls. International Journal of Multiphase Flow 28 (7), 1149-1175.

LeAL, L. G. 1992 Laminar Flow and Convective Transport Processes. Stoneham, MA: Butterworth and Heinemann.

Leshansky, AM, Lavrenteva, OM \& Nir, A 2001 Thermocapillary migration of bubbles: convective effects at low péclet number. Journal of Fluid Mechanics 443, 377-401.

Leshansky, Alexander M \& Nir, Avinoam 2001 Thermocapillary alignment of gas bubbles induced by convective transport. Journal of colloid and interface science 240, 544-551.

Limbourgfontaine, M. C., Petre, G. \& Legros, J. C. 1986 Thermocapillary movements under at a minimum of surface tension. Naturwissenschaften $\mathbf{7 3}, 360-362$.

Liu, H., VAlocchi, A.J., Zhang, Y. \& KAng, Q. 2013 Phase-field-based lattice boltzmann finite-difference model for simulating thermocapillary flows. Physical Review E - Statistical, Nonlinear, and Soft Matter Physics 87 (1).

Ma, Chen \& Bothe, Dieter 2011 Direct numerical simulation of thermocapillary flow based on the volume of fluid method. International Journal of Multiphase Flow 37 (9), 1045-1058.

Mahesri, S., Haj-Hariri, H. \& Borhan, A. 2014 Effect of interface deformability on thermocapillary motion of a drop in a tube. Heat and Mass Transfer 50 (3), 363-372.

Mazouchi, Ali \& Homsy, G. M. 2000 Thermocapillary migration of long bubbles in cylindrical capillary tubes. Physics of Fluids (1994-present) 12 (3), 542-549.

Mazouchi, Ali \& Homsy, G. M. 2001 Thermocapillary migration of long bubbles in polygonal tubes. i. theory. Physics of Fluids (1994-present) 13 (6), 1594-1600.

Mcgillis, W. R. \& Carey, V. P. 1996 On the role of marangoni effects on the critical heat flux for pool boiling of binary mixtures. Trans. ASME J. Heat Transfer 118, 103-109.

Merritt, Randy M., Morton, David S. \& Subramanian, R. Shankar 1993 Flow structures in bubble migration under the combined action of buoyancy and thermocapillarity. Journal of Colloid and Interface Science 155 (1), 200-209.

Meyyappan, M \& Subramanian, R. Shankar 1987 Thermocapillary migration of a gas bubble in an arbitrary direction with respect to a plane surface. Journal of Colloid and Interface Science 115 (1), 206-219.

Naнme, R. 1940 Beiträge zur hydrodynamischen theorie der lagerreibung. Ingenieur-Archiv 11, 191-209.

Nas, S., Muradoglu, M. \& Tryggvason, G. 2006 Pattern formation of drops in thermocapillary migration. International Journal of Heat and Mass Transfer 49 (1314), 2265-2276.

NAs, S. \& Tryggvason, G. 2003 Thermocapillary interaction of two bubbles or drops. International Journal of Multiphase Flow 29 (7), 1117-1135.

Petre, G. \& Azouni, M. A. 1984 Experimental evidence for the minimum of surface tension with temperature at aqueous alcohol solution air interfaces. J. Colloid Interface Sci. 98, 261-263.

Popinet, StÉphane 2003 Gerris: a tree-based adaptive solver for the incompressible euler equations in complex geometries. Journal of Computational Physics 190, 572-600. 
S. Ahmed, = \& CArey, V. P. 1999 Effects of surface orientation on the pool boiling heat transfer in water/2-propanol mixtures. Trans. ASME J. Heat Transfer 121, 80-88.

Savino, R., Cecere, A. \& Paola, R. Di 2009 Surface tension driven flow in wickless heat pipes with self-rewetting fluids. Int. J. Heat Fluid Flow 30, 380-388.

Savino, R., Cecere, A., Vaerenbergh, S. Van, Y.Abe, G.Pizzirusso, W.Tzevelecos, M.Mojahed \& Q.GAland 2013 Some experimental progresses in the study of the selfrewetting fluids for the selene experiment to be carried in the thermal platform 1 hardware. Acta Astronautica 89, 179-188.

Subramanian, R. Shankar 1981 Slow migration of a gas bubble in a thermal gradient. AIChE Journal 27 (4), 646654.

Subramanian, R. Shankar 1983 Thermocapillary migration of bubbles and droplets. Advances in Space Research 3 (5), 145-153.

Subramanian, R. S. 1992 The motion of bubbles and drops in reduced gravity. In Transport Processes in Drops, Bubbles and Particles (ed. R. D. Chhabra \& D. Dekee), pp. 1-41. London: Hemisphere.

Subramanian, R. S., Balasubramaniam, R. \& Wozniak, G. 2002 Fluid mechanics of bubbles and drops. In Physics of Fluids in Microgravity (ed. R. Monti), pp. 149-177. London: Taylor and Francis.

Sussman, Mark \& Smereka, Peter 1997 Axisymmetric free boundary problems. Journal of Fluid Mechanics 341, 269-294.

Suzuki, K., Nakano, M. \& Iтон, M. 2005 Subcooled boiling of aqueous solution of alcohol. In Proceedings of the 6th KSME-JSME Joint Conference on Thermal and Fluid Engineering Conference, pp. 21-23.

Tripathi, M. K., Sahu, K. C. \& Govindarajan, R. 2014 Why a falling drop does not in general behave like a rising bubble. Nature Scientific Reports 4, 4771.

Vochten, R. \& Petre, G. 1973 Study of heat of reversible adsorption at air-solution interface 2. experimental determination of heat of reversible adsorption of some alcohols. J. Colloid Interface Sci. 42, 320-327.

Welch, SAmuel WJ 1998 Transient thermocapillary migration of deformable bubbles. Journal of colloid and interface science 208 (2), 500508.

Wilson, S. K. 1993 The steady thermocapillarydriven motion of a large droplet in a closed tube. Physics of Fluids A: Fluid Dynamics (1989-1993) 5 (8), 2064-2066.

Wu, Zuo-Bing \& Hu, Wen-Rui 2012 Thermocapillary migration of a planar droplet at moderate and large marangoni numbers. Acta Mechanica 223 (3), 609-626.

Wu, Zuo-Bing \& Hu, WEN-Rui 2013 Effects of marangoni numbers on thermocapillary drop migration: Constant for quasi-steady state? Journal of Mathematical Physics 54 (2), 023102

YAriv, Ehud \& Shusser, Michael 2006 On the paradox of thermocapillary flow about a stationary bubble. Physics of Fluids (1994-present) 18 (7), 072101.

Young, N. O., Goldstein, J. S. \& Block, M. J. 1959 The motion of bubbles in a vertical temperature gradient. Journal of Fluid Mechanics 6 (03), 350-356.

Zhang, Lu, Subramanian, R. Shankar \& Balasubramaniam, R. 2001 Motion of a drop in a vertical temperature gradient at small marangoni number the critical role of inertia. Journal of Fluid Mechanics 448, 197-211.

Zhao, Jian-Fu, Li, Zhen-Dong, Li, Hui-Xiong \& Li, Jing 2010 Thermocapillary migration of deformable bubbles at moderate to large marangoni number in microgravity. Microgravity Science and Technology 22 (3), 295-303. 